signalling, in regulating intestinal epithelial apoptosis in vivo have not previously been investigated.

Aims To assess susceptibility to intestinal apoptosis and the associated molecular changes in mice with germline deletions of three individual NFKB family members.

Methods Intestinal apoptosis was induced in male c-Rel-null, NFKB1-null and NFKB2-null mice and their wild-type (C57BL/6) counterparts by $8 \mathrm{~Gy} \gamma$-irradiation ( $\mathrm{n}=6$ per group). Apoptosis was assessed on a cell positional basis from $\mathrm{H} / \mathrm{E}$ stained sections. The mRNA expression of 10 key apoptosis regulating genes in the small intestine and colon (TRAIL, Caspase12, BAK, FAS-L, FAS, p53, BCL2, BCL-XL, c-IAP2 and XIAP) was assessed by real time PCR $(n=4$ per group). Statistical comparisons were by ANOVA with Bonferroni post-hoc tests.

Results Basal small intestinal crypt apoptosis was significantly increased in NFKB2-null relative to C57BL/6 mice. In addition, small intestinal and colonic crypt apoptotic indices were both significantly

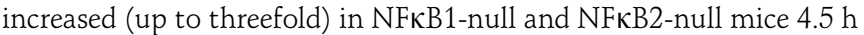
after 8Gy $\gamma$-irradiation relative to wild-type and c-Rel-null mice. Untreated NFKB1-null and NFKB2-null small intestine showed reduced mRNA expression of the anti-apoptotic genes BCL2, BCLXL, c-IAP2 and XIAP. Following irradiation, NFאB1-null mice showed significant increases in the mRNA of the pro-apoptotic genes TRAIL, Caspase12 (in both small intestine and colon) and BAK (small intestine only) compared to wild-type mice. Significant increases in the mRNA of the pro-apoptotic genes Caspase12 and FAS-L were also seen in irradiated NFKB2-null small intestine and colon relative to wild-type mice.

Conclusion c-Rel expression does not appear to regulate susceptibility to intestinal epithelial apoptosis in vivo. NFKB1 and NFKB2 deletion both caused increased susceptibility to intestinal apoptosis and this was associated with altered expression of TRAIL, Caspase12, BAK and FAS-L. These NFKB family members may therefore also regulate the susceptibility of intestinal epithelia to other consequences of DNA damage such as cancer.

Competing interests None declared.

\section{OC-020 RECURRENCE AFTER RADIOFREQUENCY ABLATION FOR BARRETT'S RELATED HIGH GRADE DYSPLASIA IS DUE TO PERSISTENCE OF EPITHELIAL MUTATIONS}

doi:10.1136/gutjnl-2012-302514a.20

${ }^{1} \mathrm{~S}$ Zeki, ${ }^{* 2} \mathrm{~L}$ Lovat, ${ }^{2} \mathrm{R}$ Haidry, ${ }^{3} \mathrm{H}$ Barr, ${ }^{4} \mathrm{~N}$ Shepherd, ${ }^{5} \mathrm{M}$ Rodriguez-Justo, ${ }^{6} \mathrm{~N}$ Wright, ${ }^{6} \mathrm{~S}$ McDonald, ${ }^{5} \mathrm{M}$ Novelli. ${ }^{1}$ Department of Digestive Diseases, Blizard Institute, London, UK; ${ }^{2}$ Department of Gastroenterology, UCLH, London, UK; ${ }^{3}$ Department of Surgery, Gloucester Royal Hospital, Gloucester, UK; ${ }^{4}$ Department of Histopathology, Gloucester Royal Hospital, Gloucester, UK; ${ }^{5}$ Department of Histopathology, UCLH, London, UK; ${ }^{6}$ Centre for Digestive Diseases, Blizard Institute, London, UK

Introduction Radiofrequency ablation (RFA) is a relatively new endoscopic method for ablation of high grade dysplasia (HGD) and intramucosal adenocarcinoma (IMC) in Barrett's oesophagus. Clinical trials have shown enduring eradication of these pathologies. ${ }^{1}$ However, some patients develop recurrent dysplasia or cancer. ${ }^{1}$ The reason for this is unknown. The aim of the study was to investigate whether this is related to the persistence of known cancer driving mutations after radiofrequency ablation.

Methods Patient records were searched for patients with recurrent HGD or IMC after RFA. Biopsies and endoscopic mucosal resection (EMR) specimens were available before and after RFA for each case. Whole biopsies underwent nested polymerase chain reaction (PCR) sequencing for mutations commonly implicated in progression to adenocarcinoma (TP53, CDKN2A, K-ras).

Results Tissue was obtained for six patients before and after RFA. All patients were male (mean age $67 \mathrm{SD} \pm 2$ ). Samples from five patients contained detectable mutations. In $3 / 5$ patients, the same mutation was found in material taken before RFA as after (all TP53 mutations). The indication for RFA in all three patients was HGD; two of the patients developed IMC after RFA. In these two cases laser microdissection was performed on the post-RFA EMR samples. PCR revealed the pre RFA mutation to also be present throughout the IMC specimen. Dysplastic tissue adjacent to the IMC contained a mixture of crypts that were either wild type for the mutation in the IMC or contained the mutation indicating that the IMC was a monoclonal outgrowth and that the persistent mutation was driving the development of the recurrence. Furthermore, in all three cases, PCR of a further specimens (performed at a later time point to the first post-RFA EMR), revealed the same mutation as the initial biopsy before RFA, and the first post-RFA EMR.

Conclusion The recurrence of dysplasia and cancer after RFA is likely to be due to failure to remove persistent, cancer driving mutations. Further work will need to be done to assess whether this is because of technical errors, or related to specific problems such as "buried Barrett's".

\section{Competing interests None declared.}

\section{REFERENCE}

1. Shaheen $\mathbf{N}$, Overholt B, Sampliner RE. Durability of radiofrequency ablation in barrett's esophagus with dysplasia. Gastroenterology 2011:141:460-8.

\section{OC-021 A NOVEL SUBSET OF FUNCTIONAL IL-10 SECRETING CD8 REGULATORY T CELLS INFILTRATE HUMAN HEPATOCELLULAR CARCINOMA}

doi:10.1136/gutjnl-2012-302514a.21

K K Li, ${ }^{*}$ S Wards, S Curbishley, D Adams. Centre for Liver Research, NIHR Biomedical Research Unit, University of Birmingham, Birmingham, UK

Introduction Tumour specific effector T-cells can be detected in the blood and tumours of patients with hepatocellular carcinoma but fail to mount effective immune responses. Attempts to amplify antitumour immune responses using immunotherapy show promise, but are hampered by the presence of suppressive regulatory T-cells (Tregs) that inhibit anti-tumour immune responses. Tregs are crucial in the maintenance of immune homeostasis and in the prevention of auto-reactive immune response but in the context of cancer they can suppress beneficial anti-tumour immunity leading to tumour progression. A novel subset of CD8 expressing Tregs has recently been described and we now report the presence of such cells in human hepatocellular carcinoma and define their functional and homing properties.

Methods Fresh tissue from hepatocellular carcinoma and matched distal non-involved tissue was obtained from patients undergoing liver resection or transplantation at the Queen Elizabeth Hospital, Birmingham after informed consent. Liver-derived T-cells were isolated and phenotyped using multi-colour flow cytometry including intracellular cytokine staining. $\mathrm{CD} 8^{+}$Tregs were isolated using a MoFlow cell sorter for functional assays. Distribution of $\mathrm{CD} 8^{+}$Tregs was investigated by immunohistochemistry and immunofluorescence.

Results The percentage of $\mathrm{CD}^{+}$Tregs (defined as $\mathrm{CD} 8{ }^{+} \mathrm{CD} 25^{\text {high }} \mathrm{CD} 127^{\text {low }} \mathrm{FoxP} 3^{+}$) infiltrating hepatocellular carcinoma tumours was significantly greater compared with matched non-involved liver. Tumour-derived $\mathrm{CD}^{+}$Treg isolated by Mo-Flo sorting suppressed allogeneic effectors cells in vitro and secreted interleukin-10 (IL-10). In contrast T-cell interferon- $\gamma$ (IFN- $\gamma$ ) production was decreased within the tumour compared with matched non-involved liver. The chemokine receptor CXCR3 which is involved in T-cell recruitment to the inflamed liver was highly expressed on tumour-derived $\mathrm{CD} 8^{+}$Tregs.

Conclusion A novel subset of functional IL-10 secreting $\mathrm{CD} 8^{+}$Tregs may suppress anti-tumour immunity in hepatocellular carcinoma. 